\title{
Criteria for the Existence of Common Points of Spectra of Several Operator Pencils
}

\author{
R.M. Dzhabarzadeh \\ Institute of Mathematics and Mechanics, National Academy of Science of Azerbaijan, Azerbaijan
}

Copyright $@ 2015$ Horizon Research Publishing All rights reserved.

\begin{abstract}
In this paper we present two criteria for the existence of common eigen values of several operator pencils having discrete spectrum. One of the given criteria is proved by using analogs of resultant for several operator pencils; proof of the other criterion requires the use of the results of the multiparameter spectral theory. In both cases the number of operator pencils is finite, operator pencils act, generally speaking, in different Hilbert spaces.
\end{abstract}

Keywords Operator Pencils, Resultant of Two Operator Pencils, Hilbert Space, Common Eigenvalue, Kernel of Operator

\section{Introduction}

The definition of abstract analogue of resultant of two operator pencils in one parameter is given in [2] and [10] with the help of definitions of tensor product of spaces and tensor product of operators. When the operator pencils have the identical degree concerning parameter concept of resultant has been given in work of Khayniq [9], for operator pencils, generally speaking, with the different orders of parameter, the abstract analog of a resultant is studied by Balinskii [2].

Let be

$$
\begin{aligned}
& A(\lambda)=A_{0}+\lambda A_{1}+\lambda^{2} A_{2}+\ldots+\lambda^{n} A_{n}, \\
& B(\lambda)=B_{0}+\lambda B_{1}+\lambda^{2} B_{2}+\ldots+\lambda^{m} B_{m}
\end{aligned}
$$

two operator pencils depending on the same parameters $\lambda$ and acting, generally speaking, in various Hilbert spaces $H_{1}, H_{2}$, correspondingly.

$\operatorname{Re} s(A(\lambda), B(\lambda))$ is the resultant of two operator pencils $A(\lambda)$ and $B(\lambda)$. It is presented by the matrices (2) and acts in the space $\left(H_{1} \otimes H_{2}\right)^{n+m}$ - direct sum of $n+m$ copies of tensor product of space $H_{1} \otimes H_{2}$.
$\operatorname{Re} s(A(\lambda), B(\lambda))=\left(\begin{array}{cccccc}A_{0} \otimes E_{2} & A_{1} \otimes E_{2} & \ldots & A_{n} \otimes E_{2} & \ldots & 0 \\ . & . & \ldots & . & \ldots & . \\ 0 & 0 & \ldots A_{0} \otimes E_{2} & A_{1} \otimes E_{2} & \ldots & A_{n} \otimes E_{2} \\ E_{1} \otimes B_{0} & E_{1} \otimes B_{1} & \ldots & E_{1} \otimes B_{m} & . . & 0 \\ . & . & \ldots & . & \ldots & . \\ . & . & \ldots E_{1} \otimes B_{0} & E_{1} \otimes B_{1} & \ldots & E_{1} \otimes B_{m}\end{array}\right)$

In a matrix $\operatorname{Re} s\left(A_{1}(\lambda), A_{2}(\lambda)\right)$ the number of rows with operators $A_{i}$ is equal to leading degree of parameter $\lambda$ in pencil $B(\lambda)$, that is $m$, the number of rows in matrix $\operatorname{Re} s\left(A_{1}(\lambda), A_{2}(\lambda)\right)$ with operators $B_{i}$ coincides with the leading degree of parameter $\lambda$ in pencil $A(\lambda)$, that is $n$.

In [2], [10] operator $\operatorname{Re} s(A(\lambda), B(\lambda))$ is named by abstract analog of a resultant for operator pencils (1). Value of a resultant $\operatorname{Re} s(A(\lambda), B(\lambda))$ is equal to its formal decomposition wherein each term of decomposition is tensor product of operators.

Theorem 1. ([2],[10]). Let the following conditions satisfy:

a) Operators $A_{i}$ and $B_{i}$ are bounded and one of them has bounded inverse.

b) Spectra of operator pencils $A(\lambda)$ and $B(\lambda)$ are

discrete set.

Then the pencils (1) have a common eigenvalue if and only if $\operatorname{Ker} \operatorname{Re} s(A(\lambda), B(\lambda)) \neq\{\theta\}$.

In the case $m=n$ in (1) Theorem1 is proved in [10], at arbitrary whole meanings $m, n$ Theorem1 is proved in [2].

Operator (2) is the generalization of Resultant

$\operatorname{Re} s(f, g)=\left(\begin{array}{cccccccccc}a_{n} & a_{n-1} & \ldots & a_{1} & a_{0} & 0 & \ldots & \ldots & 0 & 0 \\ 0 & a_{n} & \ldots & a_{2} & a_{1} & a_{0} & \ldots & \ldots & 0 & 0 \\ . & . & \ldots & . & . & . & \ldots & \ldots & . & . \\ . & . & \ldots & . & . & . & \ldots & \ldots & . & . \\ 0 & 0 & \ldots & 0 & 0 & a_{n} & \ldots & \ldots & a_{1} & a_{0} \\ b_{m} & b_{m-1} & \ldots & b_{3} & b_{2} & b_{1} & b_{0} & \ldots & 0 & 0 \\ 0 & b_{m} & \ldots & b_{4} & b_{3} & b_{2} & b_{1} & b_{0} \ldots & 0 & 0 \\ & . & \ldots & . & . & . & . & \ldots & . & . \\ & . & \ldots & . & . & . & . & \ldots & . & . \\ & 0 & 0 & 0 & 0 & b_{m} & b_{m-1} & \ldots & b_{1} & b_{0}\end{array}\right)$ 
constructed for polynomials

$$
\begin{array}{ll}
f(x)=a_{n} x^{n}+a_{n-1} x^{n-1}+\ldots+a_{0}, & a_{n} \neq 0 ; \\
g(x)=b_{m} x^{m}+b_{m-1} x^{m-1}+\ldots+b_{0}, & b_{m} \neq 0 ;
\end{array}
$$

At the proof of the second criterion of existence of common eigenvalue of several operator pencils in Hilbert space we essentially use the results of multiparameter spectral theory $[1,3,5,11]$ and also the notion of abstract analog of Cramer's determinants.

\section{Criterion for the existence of common eigenvalues of several operator pencils in Hilbert space}

1. Let now we have $n$ the operator pencils depending on the same parameter $\lambda$

$$
\left\{\begin{array}{l}
B_{i}(\lambda)=B_{0, i}+\lambda B_{1, i}+\ldots+\lambda^{k_{i}} B_{k_{i}, i} \\
i=1,2, \ldots, n
\end{array}\right.
$$

when $B_{i}(\lambda)$ be the operator pencils acting in Hilbert space $H_{i}$, correspondingly. Without loss of generality we suppose $k_{1} \geq k_{2} \geq \ldots \geq k_{n}$.

Definition1. $[1,3,11] B_{s, i}^{+}$-the operator induced by operator $B_{s, i}$, acting from the space $H_{i}$ into tensor product space $H=H_{1} \otimes \ldots \otimes H_{n}$ and is defined the following way: on decomposable tensor $x=x_{1} \otimes \ldots \otimes x_{n}$ : $B_{s, i}^{+} x=x_{1} \otimes \ldots \otimes x_{i-1} \otimes B_{s, i} x_{i} \otimes x_{i+1} \otimes \ldots \otimes x_{n} \quad, \quad$ on other elements of space $H=H_{1} \otimes \ldots \otimes H_{n}$ operator $B_{s, i}^{+}$is defined on linearity and continuity.

Introduce the operators $R_{i}(i=1, \ldots, n-1) \quad$ in space $H^{k_{1}+k_{2}}$ (the direct sum of $k_{1}+k_{2}$ copies of tensor product $H=H_{1} \otimes \ldots \otimes H_{n}$ of spaces $\left.H_{1}, H_{2}, \ldots, H_{n}\right)$ by means of the operational matrices

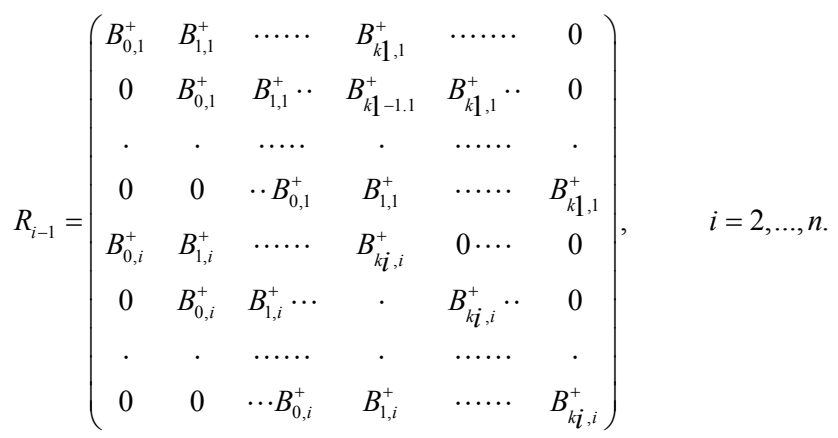

In the matrix $R_{i-1}$ the number of lines with operators $B_{s, 1}^{+}, s=0,1, \ldots, k_{1}$ is equal to $k_{2}$ and the number of lines with operators $B_{s, i}^{+}, s=0,1, \ldots, k_{i}$ is equal to $k_{1} . R_{i-1}$ are the operators in the space $H^{k_{1}+k_{2}}$ representing by the matrices in (6). (3.13)

We shall designate $\sigma_{p}\left(B_{i}(\lambda)\right)$ the set of eigenvalues of an operator $B_{i}(\lambda)$.

Theorem 2. [4] Let the operator $B_{k_{1}}$ has inverse. $\bigcap_{i=1}^{n} \sigma_{p}\left(B_{i}(\lambda)\right) \neq\{\theta\}$ iff $\bigcap_{i=1}^{n} \operatorname{Ker} R_{i} \neq\{\theta\}$.

Proof of Theorem 2. Necessity. We suppose, that pencils $B_{i}(\lambda)$ have a common eigen- value $\lambda^{0}$. For everyone $i$ there are such elements $x_{i} \in H_{i}$, that $B_{i}^{+}\left(\lambda^{0}\right) x_{1} \otimes \ldots \otimes x_{n}=0, \quad i=1,2, \ldots, n$.

It is easy to see, if the element

$$
X=\left(x_{1} \otimes \ldots \otimes x_{n}, \lambda^{0} x_{1} \otimes \ldots \otimes x_{n}, \ldots,\left(\lambda^{0}\right)^{k_{1}+k_{2}-1} x_{1} \otimes \ldots \otimes x_{n}\right)
$$

in the kernel of an operator $R_{i}$ for each $i=1,2, \ldots, n-1$, then $X \in \bigcap_{i=1}^{n-1} \operatorname{Ker} R_{i}$.

Sufficiency. Let $\bigcap_{i=1}^{n-1} \operatorname{Ker} R_{i} \neq\{\theta\} \quad$ and $\quad$ an element $X=\left(\tilde{x}_{1}, \tilde{x}_{2}, \ldots, \tilde{x}_{k 1+k 2}\right) \in \bigcap_{i=1}^{n-1} \operatorname{Ker} R_{i}, \quad \tilde{x}_{i} \in H$. Then element $X$ in the kernels of operators $R_{1}, R_{2}, \ldots, R_{n-1} \quad, \quad$ i. $\quad$ e. $R_{s}\left(\tilde{x}_{1}, \widetilde{x}_{2}, \ldots, \tilde{x}_{k_{1}+k_{2}}\right)=\theta ; s=1,2, \ldots, n-1$. Expression $\bigcap_{i=1}^{n-1} \operatorname{Ker}_{i} \neq\{\theta\}$ means there is such nonzero element $\left(\sum_{i=1}^{s} x_{1, i}^{(s)} \otimes x_{2, i}^{(s)} \otimes \ldots \otimes x_{n, i}^{(s)}\right)_{s=1}^{k 1+k 2} \in\left(H_{1} \otimes \ldots \otimes H_{n}\right)^{k 1+k 2}$ that the equalities satisfy. Then an element $\left(\sum_{i=1}^{\infty} x_{1, i}^{(s)} \otimes x_{2, i}^{(s)} \otimes \ldots \otimes x_{n, i}^{(s)}\right)_{s=1}^{k 1+k 2}$ enters the kernel of Resultant of operators $B_{1}(\lambda)$ and $B^{++}\left(\lambda, \alpha_{2}, \ldots, \alpha_{n}\right)=$ $\alpha_{2} B_{2}^{++}(\lambda)+\alpha_{3} B_{3}^{++}(\lambda)+\ldots+\alpha_{n} B_{n}^{++}(\lambda)$. 


$$
\begin{aligned}
& B_{0,1}^{+} \sum_{i=1}^{s 1} x_{1, i}^{(1)} \otimes x_{2, i}^{(1)} \otimes \ldots \otimes x_{n, i}^{(1)}+\ldots+B_{k 1,1}^{+} \sum_{i=1}^{s k_{1}+1} x_{1, i}^{(k 1+1)} \otimes \ldots \otimes x_{n, i}^{(k 1+1)}=0 \\
& B_{0,1}^{+} \sum_{i=1}^{s k_{2}} x_{1, i}^{(k 2)} \otimes x_{2, i}^{(k 2)} \otimes \ldots \otimes x_{n, i}^{(k 2)}+\ldots+B_{k 1,1}^{+} \sum_{i=1}^{s k_{1}+k_{2}} x_{1, i}^{(k 1+k 2)} \otimes \ldots \otimes x_{n, i}^{(k 1+k 2)}=0 \\
& B_{0, i}^{+} \sum_{i=1}^{s 1} x_{1, i}^{(1)} \otimes x_{2, i}^{(1)} \otimes \ldots \otimes x_{n, i}^{(1)}+\ldots+B_{k i, i}^{+} \sum_{i=1}^{s k} x_{1, i}^{(k i+1)} \otimes \ldots \otimes x_{n, i}^{(k i+1)}=0 \\
& B_{0, i}^{+} \sum_{i=1}^{s k_{1}} x_{1, i}^{(k 1)} \otimes \ldots \otimes x_{n, i}^{(k 1)}+\ldots+B_{k i, i}^{+} \sum_{i=1}^{s k_{1}+k_{2}} x_{1, i}^{(k 1+k 2)} \otimes \ldots \otimes x_{n, i}^{(k 1+k 2)}=0
\end{aligned}
$$

$B^{++}\left(\lambda, \alpha_{2}, \ldots, \alpha_{n}\right)$-the operator induced to the space $H_{2} \otimes \ldots \otimes H_{n}$ by an operator $B_{i}(\lambda)$ and $\alpha_{i}(i=2,3, \ldots, n)$ arbitrary complex numbers. The Resultant of pencils $B_{1}(\lambda)$ and $B^{++}\left(\lambda, \alpha_{2}, \ldots, \alpha_{n}\right)$ acts in the direct sum of $k_{1}+k_{2}$ copies of tensor product $H_{1} \otimes \ldots \otimes H_{n}$ of spaces $H_{1}, \ldots, H_{n}$.

Further we use the known property of the elements of tensor product space. It is known that the representation of the element in tensor product space is not unique. For each element of the tensor product space there is the number coinciding with the minimal number of decomposable tensors, necessary for the representation of this element. This number is named the rank of element. If the sum decomposable tensors in the representation of element are more than the rank of this element then one-nominal components of the given element are linear dependent. Having transferred to each line of equalities (7) one decomposable tensor from left side in the right side of the this eqalities, we get, that the series standing at the left side in each equation have a rank 1 as they are equal to a decomposable tensor, standing in the right side of this equality. Thus, between one-nominal components of all terms entering into expression (7) there is a linear dependence and an element standing at the left in all equalities in (7) is a decomposable tensor.

We have that

$$
\left(\sum_{i=1}^{s} x_{1, i}^{(s)} \otimes x_{2, i}^{(s)} \otimes \ldots \otimes x_{n, i}^{(s)}\right) \in H_{1} \otimes \ldots \otimes H_{n}
$$

is decomposable vector for all values of number $S$. Using [2] we prove there is a number $\lambda$ being the common point of spectra of operators $B_{1}(\lambda)$ and $B^{++}\left(\lambda, \alpha_{2}, \ldots, \alpha_{n}\right)$ at all values $\alpha_{i}$. The last means, that $\lambda$ there is a common eigenvalue of all pencils $B_{i}(\lambda), \quad i=1,2, \ldots, n$. Theorem2 is proved.

2. Give some definitions and concepts of the theory of multiparameter operator systems in the case when the number of parameters is equal to the number of equations.

Let the linear multiparameter system in the form be:

$$
B_{k}(\lambda) x_{k}=\left(B_{0, k}+\sum_{k=1}^{n} \lambda_{k} B_{i, k}\right) x_{k}=0 \quad k=1,2, \ldots n
$$

when operators $B_{k, i}$ act in the Hilbert space $H_{i}$

Definition 2. $[1,2,11] \lambda=\left(\lambda_{1}, \lambda, \ldots, \lambda_{n}\right) \in C^{n}$ is an eigenvalue of the system (8) if there are non-zero elements $x_{i} \in H_{i}(i=1,2, \ldots, n)$ such that equalities in (8) are true, then a decomposable tensor $x=x_{1} \otimes x_{2} \otimes \ldots \otimes x_{n}$ is called the eigenvector corresponding to an eigenvalue $\lambda=\left(\lambda_{1}, \lambda, \ldots, \lambda_{n}\right) \in C^{n}$.

Definition 3 ([5], [6]).

Let $x_{0, \ldots, 0}=x_{1} \otimes x_{2} \otimes \ldots \otimes x_{n}$ be an eigenvector of the system (8), corresponding to its eigenvalue $\lambda=\left(\lambda_{1}, \lambda_{2}, \ldots, \lambda_{n}\right)$; the $x_{m_{1}, m_{2}, \ldots, m_{n}}$ is $m_{1}, m_{2}, \ldots, m_{n}$ - the associated vector (see[5]) to an eigenvector $x_{0, \ldots, 0}$ of the system (8) if there is a set of vectors $\left(x_{i_{1}, i_{2}, \ldots, i_{n}}\right) \subset H_{1} \otimes \ldots \otimes H_{n}$, satisfying to conditions

$$
\begin{gathered}
B_{0, i}^{+}(\lambda) x_{s_{1,}, s_{2}, \ldots, s_{n}}+B_{1, i}^{+} x_{s_{1}-1, s_{2}, \ldots, s_{n}}+\ldots+B_{k, i}^{+} x_{s_{1}, s_{2}, \ldots, s_{n}-1}=0 \\
. \quad x_{i_{1}, i_{2}, \ldots, i_{n}}=0 \text {, when } s_{i}<0 \\
0 \leq s_{r} \leq m_{r}, r=1, \ldots, n ; \quad i=1, \ldots, n,
\end{gathered}
$$

Indices $S_{1}, S_{2}, \ldots, S_{n}$ in element 
$\left(x_{i_{1}, i_{2}, \ldots, i_{n}}\right) \subset H_{1} \otimes \ldots \otimes H_{n}$ there are various

arrangements from set of integers on $n$ with $0 \leq s_{r} \leq m_{r}$, $r=1,2, \ldots, n$.

Definition 4. In $[1,3,11]$ for the system (8) analogue of the Cramer's determinants, when the number of equations is equal to the number of variables, is defined as follows: on decomposable tensor $x=x_{1} \otimes x_{2} \otimes \ldots \otimes x_{n}$ operators

$\Delta_{i}$ are defined with the help the matrices

$$
\sum \alpha_{i} \Delta_{i} x=\otimes
$$

$\left(\begin{array}{cccccc}\alpha_{0} & \alpha_{1} & \alpha_{2} & \cdot & \cdot & \alpha_{n} \\ B_{0,1} x_{1} & B_{1,1} x_{1} & B_{2,1} x_{1} & . & . & B_{n, 1} x_{1} \\ B_{0,2} x_{2} & B_{1,2} x_{2} & B_{2,2} x_{2} & . & . & B_{n, 2} x_{2} \\ B_{0,3} x_{3} & B_{1,3} x_{3} & B_{2,3} x_{3} & \cdot & . & B_{n, 3} x_{3} \\ \cdot & \cdot & \cdot & \cdot & . & \cdot \\ B_{0, n} x_{n} & B_{1, n} x_{n} & B_{2, n} x_{n} & . & . & B_{n, n} x_{n}\end{array}\right)$

where $\alpha_{0}, \alpha_{1}, \ldots, \alpha_{n}$ are arbitrary complex numbers. The decomposition of the determinant (10) is its formal decomposition, when all terms of decomposition are tensor products of elements. If $\alpha_{k}=1, \alpha_{i}=0, i \neq k$,then right side of (10) is equal to $\Delta_{k} x$, where $x=x_{1} \otimes x_{2} \otimes \ldots \otimes x_{n} . \quad$ On all the other elements of the space $H$ operators $\Delta_{i}$ are defined on linearity and continuity. $E_{s}(s=1,2, \ldots, n)$ is the identity operator of space $H_{s}$. Suppose that for all $x \in H, x \neq 0$, $\left(\Delta_{0} x, x\right) \geq \delta(x, x)>0, \quad \delta>0$, and all $B_{i, k}$ are selfadjoint operators in the space $H_{i}$. Inner product [.,.] is defined as follows; if $x=x_{1} \otimes x_{2} \otimes \ldots \otimes x_{m}$ and $y=y_{1} \otimes y_{2} \otimes \ldots \otimes y_{m}$ are decomposable tensors, then $[x, y]=\left(\Delta_{0} x, y\right)$, where $\left(x_{i}, y_{i}\right)$ is the inner product in the space $H_{i}$. On all the other elements of the space $H$ the inner product is defined on linearity and continuity. In space $H$ with such metric all operators $\Gamma_{i}=\Delta_{0}^{-1} \Delta_{i}$ are selfadjoint $[1,3,11]$.

We give a new criterion for the existence of common points of spectra of all operator pencils (5). Let us assume that each operator pencil in (5) has a discrete spectrum. Conditions on the operators $B_{j, k}$ remain the same. Let highest degree of parameter $\lambda$ in (5) is $m$.

In (5) we make the transformations

$$
\lambda=\lambda_{1}, \ldots, \lambda^{i}=\lambda_{i}, \ldots, i=1,2, \ldots, m .
$$

then the operator pencils (5) are written in the form

$$
B_{i}^{+}\left(\lambda_{1}, \ldots, \lambda_{m}\right) \widetilde{x}_{1}=0 \quad i=1, \ldots, k
$$

by the operator $B_{i}\left(\lambda_{1}, \ldots, \lambda_{m}\right)$, that acts in space $H_{i}$.

The equation $B_{i}^{+}\left(\lambda_{1}, \ldots, \lambda_{m}\right) \tilde{x}_{1}=0 \quad i=1, \ldots, k$ we consider together with the following equations

$$
\begin{aligned}
& \left(t_{2}+\lambda_{1} t_{0}+\lambda_{2} t_{1}\right) x_{2}=0 \\
& \left(\lambda_{1} t_{2}+\lambda_{2} t_{0}+\lambda_{3} t_{1}\right) x_{3}=0
\end{aligned}
$$

$$
\left(\lambda_{m-2} t_{2}+\lambda_{m-1} t_{0}+\lambda_{m} t_{1}\right) x_{m}=0
$$

where the operators $t_{0}, t_{1}, t_{2}$ are defined with help of the matrices

$$
t_{0}=\left(\begin{array}{ll}
0 & 1 \\
1 & 0
\end{array}\right), \quad t_{1}=\left(\begin{array}{ll}
1 & 0 \\
0 & 0
\end{array}\right), t_{2}=\left(\begin{array}{ll}
0 & 0 \\
0 & 1
\end{array}\right)
$$

In the space $R^{2}$ the equations (13) on its eigenvectors realize the connections between the parameters $\lambda_{1}, \lambda_{2}, \ldots, \lambda_{m}$ according to the requirements of (5).

We build $k$ linear multiparameter systems

$$
\begin{aligned}
& B_{i}^{+}\left(\lambda_{1}, \ldots, \lambda_{m}\right) \widetilde{x}_{1}=0 \\
& \left(t_{2}+\lambda_{1} t_{0}+\lambda_{2} t_{1}\right) x_{2}=0 \\
& \left(\lambda_{1} t_{2}+\lambda_{2} t_{0}+\lambda_{3} t_{1}\right) x_{3}=0 \\
& \left(\lambda_{m-2} t_{2}+\lambda_{m-1} t_{0}+\lambda_{m} t_{1}\right) x_{m}=0
\end{aligned}
$$

$\widetilde{x}_{1} \in H_{1} \otimes \ldots \otimes H_{k}, \quad x_{s} \in R^{2}, s=2, \ldots, m-1 ; i=1, \ldots, k$.

and introduce the space

$\overleftrightarrow{H}=H_{1} \otimes \ldots \otimes H_{k} \otimes R^{2} \otimes \ldots \otimes R^{2}$. In the tensor product $\overleftrightarrow{H}$ factor $R^{2}$ repeats $m-1$ time. Construct the analog determinants of Cramer for the linear multiparameter system (15) of the formulae

$$
\sum_{i=0}^{m} \alpha_{i} \Delta_{i}=\left(\begin{array}{cccccc}
\alpha_{0} & \alpha_{1} & \alpha_{2} & . & . & \alpha_{m} \\
B_{0, i}^{++} & B_{1, i}^{++} & B_{2, i}^{++} & . & . & B_{m, i}^{++} \\
t_{1,2}^{++} & t_{0,2}^{++} & t_{2,2}^{++} & . & . & 0 \\
0 & t_{1,3}^{++} & t_{0,3}^{++} & . & . & 0 \\
\cdot & \cdot & . & . & . & . \\
0 & 0 & 0 & . & . & t_{2, m}^{++}
\end{array}\right)
$$

When $B_{j, i}=0$ if $j>k_{i}$. All operators $B_{i, j}^{++}, t_{s, k}^{++}$in the expression (16) are induced in space $\overleftrightarrow{H}=H_{1} \otimes \ldots \otimes H_{k} \otimes R^{2} \otimes \ldots \otimes R^{2}$ by the 
operators $B_{i, j}^{+}, t_{s, k}$, correspondingly.

If operators $B_{i, k}$ are selfadjoint in space $H_{k}$ and above accepted conditions are satisfied, then all the eigenvalues of each operator

$\Gamma_{i, s}=\Delta_{0, s}^{-1} \Delta_{i, s}(s=1, \ldots, k ; i=1, \ldots, m$.$) \quad , are real$ numbers $[1,3,11]$. Suppose now that $\left(\lambda_{1}, \ldots, \lambda_{m}\right)$ is an eigenvalue of the system (15). Take two equations of the system, namely the equations

$$
\begin{aligned}
& \left(t_{2}+\lambda_{1} t_{0}+\lambda_{2} t_{1}\right) x_{2}=0 \\
& \left(\lambda_{1} t_{2}+\lambda_{2} t_{0}+\lambda_{3} t_{1}\right) x_{3}=0
\end{aligned}
$$

For eigenvector

$x_{1} \otimes \ldots \otimes x_{m}, x_{1} \in R^{2}, \ldots, x_{m-1} \in R^{2},\left(x_{1} \otimes \ldots \otimes x_{k}\right) \in H_{1} \otimes \ldots \otimes H_{k} \quad$ of the system (15) we have the following:

If $\lambda_{1} \neq 0$ and $x_{2}=\left(\alpha_{1}, \beta_{1}\right)$ is eigenvector of the first equation of (16) then

$\left(\left(\begin{array}{ll}0 & 0 \\ 0 & 1\end{array}\right)+\lambda_{1}\left(\begin{array}{ll}0 & 1 \\ 1 & 0\end{array}\right)+\lambda_{2}\left(\begin{array}{ll}1 & 0 \\ 0 & 0\end{array}\right)\right)\left(\alpha_{1}, \beta_{1}\right)=0$,

or $\lambda_{1} \beta_{1}+\lambda_{2} \alpha_{1}=0, \beta_{1}+\lambda_{1} \alpha_{1}=0, \lambda_{2} \neq 0 ; \lambda_{2}=\lambda_{1}^{2}$.

Further if $\lambda_{1} \neq 0, \lambda_{2} \neq 0, x_{n+2}=\left(\alpha_{2}, \beta_{2}\right) \neq 0$ then the equalities $\lambda_{2} \beta_{2}+\lambda_{3} \alpha_{2}=0, \lambda_{1} \beta_{2}+\lambda_{2} \alpha_{1}=0$ and $\lambda_{1} \lambda_{3}=\lambda_{2}^{2}$.Earlier we proved $\lambda_{2}=\lambda_{1}^{2}$, therefore $\lambda_{3}=\lambda_{1}^{3}$.

By analogy for other equations: if $\left(\lambda_{1}, \lambda_{2}, \ldots, \lambda_{m}\right)$ is an eigenvalue of the system (15) then $\lambda_{4}=\lambda_{1}^{4} \quad \ldots$, $\lambda_{m}=\lambda_{1}^{m}, \lambda=\lambda_{1}$ are valid.

In the space $\vec{H}=H_{1} \otimes \ldots \otimes H_{k} \otimes R^{2} \otimes \ldots \otimes R^{2}$ we build operators $\Delta_{0,1}, \ldots, \Delta_{0, k}$ (accord to the definition 4) for each of linear multiparameter system (15). If kernels of operators $\Delta_{0,1}, \ldots, \Delta_{0, k}$. are zero then (see [1],[3],[5]) the parameter $\lambda_{i}$ in (15) are separated. The formulae

$$
\begin{aligned}
& \Delta_{0, s}^{-1} \Delta_{1, s} \vec{x}_{1}=\lambda_{1} \vec{x}_{1}, \ldots ., \Delta_{0, s}^{-1} \Delta_{m, s} \vec{x}_{m}=\lambda_{m} \vec{x}_{m} ; s=1, \ldots, k \\
& \vec{x} \in \overleftrightarrow{H}=H_{1} \otimes \ldots \otimes H_{k} \otimes R^{2} \otimes \ldots \otimes R^{2}
\end{aligned}
$$

are true. So on eigenvectors of the system $\lambda_{2}=\lambda_{1}^{2}, \lambda_{3}=\lambda_{1}^{3}, \lambda_{4}=\lambda_{1}^{4}, \ldots, \lambda_{m}=\lambda_{1}^{m}, \lambda=\lambda_{1}$ and

$$
\Delta_{0,1}^{-1} \Delta_{i, 1} \vec{x}_{1}=\lambda_{1}^{i} \vec{x}_{1}, \ldots . ., \Delta_{0, k}^{-1} \Delta_{m, k} \vec{x}_{m}=\lambda_{1}^{m} \vec{x}_{m}
$$

Besides of $\lambda=\lambda_{1}$ then

$$
\Delta_{0, s}^{-1} \Delta_{i, s} \vec{x}_{i}=\lambda^{i} \vec{x}_{i}, \quad s=1,2, \ldots, k ; i=1,2, \ldots, m
$$

Theorem3. Let the spectra of the operator pencils $B_{i}(\lambda)$ contain only eigenvalues, operators $\Delta_{0, i}^{-1} \quad(i=1,2, \ldots, k)$ exist and bounded. Then $\lambda$ is a common eigenvalue of all the operators $B_{i}(\lambda)$ in (5) iff

$$
\operatorname{Ker}\left(\Delta_{1,1}-\lambda \Delta_{0,1}\right) \cap \ldots \cap \operatorname{ker}\left(\Delta_{1, k}-\lambda \Delta_{0, k}\right) \neq\{\theta\}
$$

Proof of Theorem3. Suppose that conditions of the Theorem 3are fulfilled. From [ 5] it follows: system of eigen and associated vectors of all multiparameter systems (15) and system of eigen and associated vectors of operators

$$
\Gamma_{i, 1}=\Delta_{0,1}^{-1} \Delta_{i, 1}, \ldots ., \Gamma_{i, k}=\Delta_{0, k}^{-1} \Delta_{i, k} \quad(i=1,2, \ldots, m)
$$

coincide for the each fixed meanings of number $i$. In virtue of the connections between the components of eigenvalues of the system(15) we have

$$
\begin{aligned}
\Delta_{0, s}^{-1} \Delta_{i, s} \vec{x}_{i} & =\lambda_{i} \vec{x}_{i} ; \quad \vec{x}_{i}=\vec{x} ; \quad i=1, . ., m ; \quad s=1, \ldots, k ; \quad \text { or } \\
\Delta_{0, s}^{-1} \Delta_{i, s} \vec{x} & =\lambda^{i} \vec{x} ; \quad i=1, . ., m ; \quad s=1, \ldots, k ; \quad
\end{aligned}
$$

Let $\lambda$ is the first component of eigenvalue of all systems (15). From condition

$\operatorname{Ker}\left(\Delta_{1,1}-\lambda \Delta_{0,1}\right) \bigcap \ldots \cap \operatorname{ker}\left(\Delta_{1, m}-\lambda \Delta_{0, m}\right) \neq\{\theta\} \quad$ it follows that there is the element

$$
\tilde{x}_{1} \otimes x_{2} \otimes \ldots \otimes x_{m}=\vec{x} \in \vec{H} ; \tilde{x}_{1} \in H_{1} \otimes \ldots \otimes H_{k} \quad,
$$
being the common eigenvector of all systems (15), corresponding to the common eigenvalue $\left(\lambda_{1}, \lambda_{2}, \ldots, \lambda_{m}\right)=\left(\lambda, \lambda^{2}, \ldots, \lambda^{m}\right)$.

We use the known formulae of multiparameter spectral theory $[1,3,11]$

$$
\begin{aligned}
B_{0, i}^{++}+ & B_{1, i}^{++} \Gamma_{1, i}+\ldots+B_{m, i}^{++} \Gamma_{m, i}=0, i=1.2, \ldots, k \\
& \left(t_{2,2}^{++}+t_{0,2}^{++} \Gamma_{1, i}+t_{1,2}^{++} \Gamma_{2, i}=0\right. \\
& t_{2,3}^{++} \Gamma_{1, i}+t_{0,3}^{++} \Gamma_{2, i}+t_{1,3}^{++} \Gamma_{3, i}=0 \\
& \cdots \ldots \ldots \ldots \ldots \ldots \ldots \ldots \ldots \ldots \ldots \ldots \ldots \ldots \ldots \ldots \ldots \ldots \ldots \ldots \ldots \ldots \ldots \ldots \ldots \ldots \\
& t_{2, m-1}^{++} \Gamma_{m-2, i}+t_{0, m-1}^{++} \Gamma_{m-1, i}+t_{1, m-1}^{++} \Gamma_{m, i}=0
\end{aligned}
$$

$i=1, \ldots, k$. Substituting into the first equality in all multiparameter systems (22) values of operators $\Gamma_{s, r}$ from (20),(21) and given $\vec{x}_{1}=\vec{x}_{2}=\ldots=\vec{x}_{m}=\vec{x}$ we establish that all operator pencils in (5) have a common eigenvalue.

It is true an inverse preposition. Let $\lambda$ there is a common eigenvalue of the operator pencils (5), consequently, $\left(\lambda, \lambda^{2}, \ldots, \lambda^{m}\right)$ is a eigenvalue of all systems in (15). So $\lambda$ is the eigenvalue of (15), then there is eigenvector $\vec{x}_{1}=\vec{x}_{2}=\ldots=\vec{x}_{m}=\vec{x}$ of all multiparameter system in 
(15).From [5] it follows that the system of eigen and associated vectors of multiparameter system (15) and of each equation $\left(\Delta_{1,1}-\lambda \Delta_{0,1}\right) \vec{x}=0, \ldots,\left(\Delta_{1, k}-\lambda \Delta_{0, k}.\right) \vec{x}=0$ coincide. So $\vec{x}$ is an eigenvector of (15), then $\operatorname{Ker}\left(\Delta_{1,1}-\lambda \Delta_{0,1}\right) \cap \ldots \cap \operatorname{ker}\left(\Delta_{1, k}-\lambda \Delta_{0, k}\right) \neq\{\theta\}$ Theorem3 is proved.

Application. In the case when all operators in all operator pencils (5) are the real numbers, Hilbert spaces $H_{i}=R,(i=1,2, \ldots, n)$. we have $n$ polynomials

$$
\left\{\begin{array}{l}
b_{i}(x)=b_{0, i}+b_{1, i} x+\ldots+b_{k_{i}, i} x^{k_{i}} \\
i=1,2, \ldots, n
\end{array}\right.
$$

when the variable $x$ is the parameter $\lambda$ in (5). Then the polynomials (23) have the common solution then and only then when $\bigcap_{i=1}^{n} \operatorname{Ker}_{i} \neq\{\theta\} . R_{i}$ are constructed by the formula (6), in which the operators $B_{s, i}$ are replaced by the numbers $b_{s, i}$.

\section{REFERENCES}

[1] Atkinson F.V. Multiparameter spectral theory.
Bull.Amer.Math.Soc.1968, 74, 1-271.

[2] Balinskii A.I Generation of notions of Обобщение понятия Bezutiant and Resultant DAN of Ukr. SSR, ser.ph.-math and tech. of sciences, 1980,2,pp.3-6 ( in Russian).

[3] Browne P.J. Multiparameter spectral theory. Indiana Univ. Math. J,24, 3, 1974.

[4] Dzhabarzadeh R.M. On existence of common eigen value of some operator-bundles, that depends polynomial on parameter. Baku.International Topology conference, 3-9 oct., 1987, Tez., 2, Baku, 1987, p.93

[5] Dzhabarzadeh R.M. Spectral theory of two parameter system in finite-dimensional space. Transactions of NAS Azerbaijan, v. 3-4 1998, p.12-18

[6] Dzhabarzadeh R.M. Spectral theory of multiparameter system of operators in Hilbert space, Transactions of NAS of Azerbaijan, 1-2, 1999, 33-40.

[7] Dzhabarzadeh R.M. Multiparameter spectral theory . Lambert Academic Publishing, 2012, pp. 184(in Russian)

[8] Dzhabarzadeh R.M .Nonlinear algebraic system. Lambert Academic Publishing, 2013,pp. 101 (in Russian )

[9] Dzhabarzadeh R.M. About solutions of nonlinear algebraic system in two Variables. Pure and Applied Mathematics Journal,vol. 2, No. 1, pp. 32-37, 2013

[10] Khayniq Q. Abstract analog of an eliminant of two polynomial bundles. The Functional analysis and its applications, 1977,2, issue 3, pp.94-95(in Russian).

[11] Sleeman B.D. Multiparameter spectral theory in Hilbert space. Pitnam Press, London, 1978, p.118. 